\title{
Socioeconomic risk factors for lung function decline in a general population
}

\author{
A. Johannessen*, T.M.L. Eagan\#,ף, E.R. Omenaas*, P.S. Bakke ${ }^{\#,+}$ and A. Gulsvik ${ }^{\#,+}$
}

\begin{abstract}
The aim of our study was to examine sex-specific associations between different aspects of socioeconomic status (SES) (educational level, occupational status, income) and lung function in a general adult population.
\end{abstract}

In the Hordaland County Cohort Study, 1,644 subjects aged 26-82 yrs at baseline answered questionnaires and performed post-bronchodilator spirometry both in 1996-1997 and in 20032006. We performed adjusted linear regression analysis on the effect of SES on decline in forced experimental volume in $1 \mathrm{~s}$ (FEV1), forced vital capacity (FVC) and FEV $1 / F V$.

Mean annual decline in FEV 1 from baseline to follow-up was $57 \mathrm{~mL}$ (SE 1.3) and $48 \mathrm{~mL}$ (SE 1.0) for males and females, respectively. Males had a larger decline in FVC than females, while females had a larger decline in FEV 1 /FVC. Lower education and low occupational status were associated with larger male lung function decline. SES did not affect female lung function decline. However, marital status was a significant predictor; unmarried females had less decline than both married and widowed females in both FEV 1 (adjusted mean annual difference $8 \mathrm{~mL}$ and $16 \mathrm{~mL}$ ) and FVC (adjusted mean annual difference $8 \mathrm{~mL}$ and $18 \mathrm{~mL}$ ).

Low SES was associated with increased lung function decline in males. For females, marital status was more important.

KEYWORDS: Asthma, chronic obstructive pulmonary disease, epidemiology, respiratory function, risk factor, spirometry

ow socioeconomic status (SES) is inversely related to burden of illness and mortality rates from many diseases, such as coronary heart disease, different types of cancer and respiratory diseases [1-3]. The joint guidelines from the American Thoracic Society (ATS) and the European Respiratory Society present SES as a risk factor for chronic obstructive pulmonary disease along with other known factors such as smoking, environmental pollution and occupational exposure to dust or gas [4].

Several studies have shown that there is an association between low SES and impaired lung function [2, 5-9]. This association is also significant after adjusting for common confounders like smoking habits and occupational exposures. It is likely that low SES is associated with further lifestyle factors that have a negative effect on lung function, such as air pollution, unhealthy diet, passive smoking and indoor climate [10-12]

SES is a multidimensional concept consisting of at least educational level, income and occupational status [13]. Little is known with regard to how associations between SES and pulmonary health differ over time and between males and females. Of the few longitudinal studies with forced expiratory volume in $1 \mathrm{~s}$ (FEV1) as outcome, one study analysed hospital admissions [2], one study examined lung function decline of young adults only [6], one study had only male participants [8] and one study analysed mortality [9]. More information on aspects of SES and on sex differences may bring us closer to an understanding of the underlying mechanisms in the associations between SES and pulmonary health. Also marital status is an important aspect when examining such sex differences in morbidity [13]. It has been suggested that marriage may have different health effects for females and males, in that marriage protects males more than females from both overall and cause-specific mortality [14]. Thus, it is important to adjust for marital status when examining the relationship between SES and pulmonary health.

The main objective of the present study was to examine sex-specific associations between different
AFFILIATIONS

${ }^{\star}$ Centre for Clinical Research,

\#Dept of Thoracic Medicine,

Haukeland University Hospital, and + Institute of Medicine, University of Bergen, Bergen, Norway.

'Division of Physiology, Dept of Medicine, University of California, San Diego, CA, USA.

CORRESPONDENCE

A. Johannessen

Centre for Clinical Research

Haukeland University Hospital 5021 Bergen

Norway

E-mail: ane.johannessen@

helse-bergen.no

Received:

Nov 232009

Accepted after revision:

Jan 312010

First published online:

Feb 112010 
aspects of SES and lung function decline in a general adult population. Aspects of SES analysed in this study were income, educational level and occupational status.

\section{METHODS}

The present study is based on data from the first (1996-1997) and second (2003-2006) follow-up surveys in the Hordaland County Cohort Study (HCCS) [15-17]. Briefly, the HCCS initially comprised 3,181 participants from Bergen and 11 surrounding municipalities in 1985, of whom 2,358 performed acceptable spirometry in 1996-1997. Of these subjects 2,250 were residing in Hordaland and were invited to a follow-up in 2003-2006. Altogether $74 \%(1,664$ out of 2,250) of those invited performed an acceptable spirometry. Predictors for loss to follow-up were low educational level and low income for females, and low income and being married or divorced for males (see online supplementary material, table E1). In the remainder of this paper, the 1996-1997 survey will be referred to as "baseline", and the 2003-2006 survey will be referred to as "follow-up". Informed consent was obtained from each participant prior to each study phase, and the study was approved by the Regional Committee of Medical Research Ethics (Bergen, Norway).

Both surveys included extensive questionnaires and clinical examinations. Additional detail on the spirometric tests is provided in online supplementary data. The highest forced vital capacity (FVC) and FEV1 values of three trials were used in the analyses. Annual decline in FEV1, FVC and FEV1/FVC was defined as the highest 1996-1997 measurement minus the highest 2003-2006 measurement, divided by follow-up time. Mean follow-up time was 6.8 yrs with SD of 0.7 yrs.

Subjects were classified as persistent never-smokers, persistent ex-smokers, quitters, beginners and persistent smokers according to self-reported daily smoking habits in 1996-1997 and 2003-2006. All other covariates analysed in the present study were registered at baseline. Standing height without shoes was measured to the nearest centimetre at the clinical examination. 1 pack-yr was defined as 20 cigarettes a day for $1 \mathrm{yr}$. Marital status was linked from the national tax registry, and was categorised into married, unmarried, widowed and divorced.

The highest completed educational level was classified into: 1) 9 yrs of compulsory school; 2) continuation school, secondary school or technical school; and 3) college or university [18]. The participants registered their current occupation in selfadministered questionnaires. We then recoded the occupations manually into the occupational status categories white-collar high level (e.g. scientists, teachers, medical doctors), whitecollar medium level (e.g. engineers, nurses, pilots), white-collar low level (e.g. secretaries, cooks, police officers), blue-collar high level (skilled manual labour, e.g. carpenters, painters, blacksmiths) and blue-collar low level (unskilled manual labour, e.g. window cleaners, shop salespersons, waitresses) using the internationally renowned five-category EGP (Eriksson-Goldthorpe Portocarero) scheme [19]. People not working for various reasons (the majority of whom were retired) were classified as "others". Income data for each subject were linked from the national tax registry. Annual personal income in 1996-1997 was categorised based on population tertiles.
Analyses were performed using Stata Statistical Software 10.0 (StataCorp, College Station, TX, USA). Initially, we tested for interactions between sex and risk factors for lung function decline. Sex interacted significantly with age and height for FEV1/FVC decline $(\mathrm{p}<0.01)$, and borderline significantly $(\mathrm{p}<0.05$ but $>0.01)$ with marital status (for FVC decline), income (for FEV1/FVC decline) and occupational exposure to dust or gas (for FVC decline). We also performed interaction analyses of marital status/SES factors and smoking habits. Smoking interactions involving marital status, educational level and occupational status were not significant for any sex. Only one interaction was significant; male smokers with low income had larger FEV1/FVC decline than male smokers with high income. Interaction analyses of occupational exposure to dust or gas and occupational status with regard to lung function decline showed no significant interactions. To enable focus on sex-specific associations between different aspects of SES and lung function, the main analyses in our study were stratified by sex. All analyses were adjusted for age, height, smoking habits, occupational exposure to dust or gas and marital status. All p-values were two-sided, and values below 0.05 were considered statistically significant. Educational level, occupational status and income were analysed as risk factors for decline in FEV1, FVC and FEV1/FVC using linear regression analysis. Sex differences in baseline characteristics as well as in lung function decline were analysed using twogroup mean comparison test (unpaired t-test) for continuous variables and Chi-squared test for categorical variables. Oneway ANOVA with Bonferroni tests was performed to compare mean decline in FEV1, FVC and FEV1/FVC in subcategories of education, occupational status and income.

\section{RESULTS}

\section{Study population characteristics}

Of the 1,644 participants in this study, 49\% were females (table 1). Mean age at baseline was 48 yrs (SD 13 yrs), with females being slightly older than males $(p<0.05)$. More females than males were never-smokers (44\% versus $33 \%$, respectively; $\mathrm{p}<0.01)$. Among ever-smokers, females had fewer pack-yrs than males $(\mathrm{p}<0.01)$. Approximately half of the study population had experienced occupational exposure to dust or gas, although the exposure was much more prevalent among males than females $(64 \%$ and $31 \%$, respectively; $\mathrm{p}<0.01)$.

The majority of the population had intermediate education, and there were no significant sex differences in educational level. More males had high occupational status, and more females had low occupational status $(p<0.01)$. Mean annual personal income at baseline was 238,000 Norwegian kroner, significantly higher for males than females $(p<0.01)$. A majority of the population was married. Among those who were not married, more males were unmarried and more females were widowed or divorced $(p<0.01)$.

\section{Baseline and follow-up lung function by SES factors and marital status}

Mean baseline FEV1/FVC was $81 \%$, with females having a higher ratio than males $(\mathrm{p}<0.01$; table 2$)$. Lower education, low occupational status and low income were all associated with cross-sectional lung function at both baseline and follow-up for males ( $<<0.05$; see tables E2 and E4 in the online supplementary 


\begin{tabular}{|c|c|c|c|c|c|}
\hline TABLE 1 & \multicolumn{5}{|c|}{$\begin{array}{l}\text { Baseline characteristics by sex of participants in the first and second follow-up in the Hordaland County Cohort Study } \\
\text { subjects who answered questionnaires and performed post-bronchodilator spirometry at both baseline in 1996-1997 } \\
\text { and follow-up in 2003-2006 }\end{array}$} \\
\hline \multicolumn{2}{|l|}{ Age yrs } & $48.0 \pm 13.3$ & $48.7 \pm 13.4$ & $47.4 \pm 13.1$ & 0.039 \\
\hline \multicolumn{2}{|l|}{ Height m } & $1.72 \pm 0.09$ & $1.65 \pm 0.06$ & $1.78 \pm 0.07$ & $<0.001$ \\
\hline \multicolumn{2}{|c|}{ Persistent never-smokers } & $607(37)$ & $336(42)$ & $271(33)$ & \\
\hline \multicolumn{2}{|c|}{ Persistent ex-smokers } & $383(24)$ & $153(19)$ & $230(28)$ & \\
\hline \multicolumn{2}{|c|}{ Quitters } & $156(10)$ & $78(10)$ & $78(9)$ & \\
\hline \multicolumn{2}{|l|}{ Beginners } & $50(3)$ & $26(3)$ & $24(3)$ & \\
\hline \multicolumn{2}{|c|}{ Persistent smokers } & $426(26)$ & $203(26)$ & $223(27)$ & \\
\hline \multicolumn{2}{|c|}{ Occupational dust exposure $^{+}$} & $769(48)$ & $248(31)$ & $521(64)$ & $<0.001$ \\
\hline \multicolumn{2}{|c|}{ Occupational status ${ }^{f}$} & & & & $<0.001$ \\
\hline \multicolumn{2}{|c|}{ White-collar high level } & $209(13)$ & $40(5)$ & $169(20)$ & \\
\hline \multicolumn{2}{|c|}{ White-collar medium level } & $333(20)$ & $186(23)$ & $147(18)$ & \\
\hline \multicolumn{2}{|c|}{ White-collar low level } & $199(12)$ & $167(20)$ & $32(4)$ & \\
\hline \multicolumn{2}{|c|}{ Blue-collar high level } & $187(12)$ & $15(2)$ & $172(21)$ & \\
\hline \multicolumn{2}{|c|}{ Blue-collar low level } & $414(25)$ & $243(30)$ & $171(20)$ & \\
\hline \multicolumn{2}{|c|}{ Others ${ }^{\# \#}$} & $298(18)$ & $159(20)$ & $139(17)$ & \\
\hline \multicolumn{2}{|c|}{ Income (Norwegian kroner $\times 1000$ ) } & $238 \pm 400$ & $176 \pm 103$ & $299 \pm 546$ & \\
\hline \multicolumn{2}{|c|}{ Income } & & & & $<0.001$ \\
\hline \multicolumn{2}{|c|}{ Low income } & $454(28)$ & $340(42)$ & $114(14)$ & \\
\hline \multicolumn{2}{|c|}{ Medium income } & $558(34)$ & $316(39)$ & $242(29)$ & \\
\hline
\end{tabular}

Data are presented as mean $\pm \mathrm{SD}$ or $\mathrm{n}(\%)$, unless otherwise indicated. ${ }^{*}$ : $\mathrm{p}$-values are from the two-group mean comparison test (unpaired t-test) for continuous variables and Chi-squared tests for categorical variables; ": information on smoking habits from baseline to follow-up was missing for 14 females and eight males; ${ }^{+}$: information on

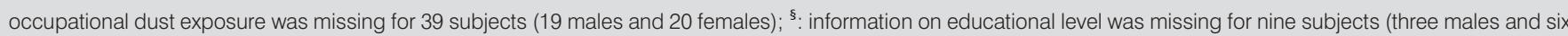

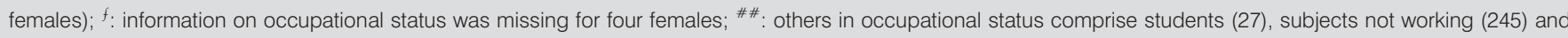
subjects not classifiable for other reasons (26).

material). For females, low occupational status was associated with lower lung function at baseline and at follow-up. In addition, married females had lower FEV1/FVC ratio than unmarried females at both points in time $(\mathrm{p}<0.05$; see tables E3 and E5 in the online supplementary material).

\section{Decline in lung function by SES factors and marital status}

Mean annual decline in lung function was $52 \mathrm{~mL}$ (SE $0.8 \mathrm{~mL}$ ) FEV1, $40 \mathrm{~mL}$ (SE $1.0 \mathrm{~mL}$ ) FVC, and 0.55\% FEV1/FVC (SE 0.02\%) (table 2). There were significant sex differences in lung function decline. Males had a larger decline in both FEV1 and FVC than females, while females, conversely, had a larger decline in FEV1/FVC $(\mathrm{p}<0.01)$.

Univariate ANOVA analyses showed no significant associations between socioeconomic risk factors and marital status, and male decline in FEV1 and FVC. For FEV1/FVC in males, however, educational level, occupational status and income was associated with larger decline $(\mathrm{p}<0.05$; see table E6 in the online supplementary material and fig. 1). Regarding SES, similar univariate results were observed for females (see table E7 in the online supplementary material and fig. 2). There was no association with female decline in FEV1 or FVC, while both educational level and occupational status were significantly associated with decline in FEV1/FVC $(\mathrm{p}<0.05)$. However, in females, marital status was associated with differences in mean FEV1 and FVC decline $(p<0.05$; see table E7 in the online supplementary material and fig. 2). Unmarried females had less decline in FEV1 and FVC than married and widowed females $(p<0.05)$. 


\begin{tabular}{|c|c|c|c|c|}
\hline \multirow[t]{2}{*}{ TABLE 2} & \multirow[b]{2}{*}{$\begin{array}{c}\text { Total } \\
\text { subjects }\end{array}$} & \multirow[b]{2}{*}{ Females } & \multirow[b]{2}{*}{ Males } & \multirow[b]{2}{*}{$p$-value ${ }^{t}$} \\
\hline & & & & \\
\hline Baseline FEV1 L & $3.42 \pm 0.02$ & $2.88 \pm 0.02$ & $3.95 \pm 0.03$ & $<0.001$ \\
\hline Baseline FVC L & $4.23 \pm 0.03$ & $3.51 \pm 0.02$ & $4.94 \pm 0.03$ & $<0.001$ \\
\hline Baseline FEV $1 /$ FVC \% & $81 \pm 0.2$ & $82 \pm 0.2$ & $80 \pm 0.2$ & $<0.001$ \\
\hline FEV1 decline $\mathrm{mL}$ & $52 \pm 0.8$ & $48 \pm 1.0$ & $57 \pm 1.3$ & $<0.001$ \\
\hline FVC decline $\mathrm{mL}$ & $40 \pm 1.0$ & $36 \pm 1.2$ & $44 \pm 1.7$ & $<0.001$ \\
\hline FEV $1 / F V C$ decline $\%$ & $0.55 \pm 0.02$ & $0.60 \pm 0.02$ & $0.50 \pm 0.02$ & 0.003 \\
\hline
\end{tabular}

Data are presented as mean $\pm \mathrm{SE}$, unless otherwise indicated. FEV1: forced expiratory volume in $1 \mathrm{~s}$; FVC: forced vital capacity. ${ }^{*}$ : $p$-values are from twogroup mean comparison test (unpaired t-test) for continuous variables. $n=1644$.

Multivariate analysis of lung function decline by SES factors Sex-specific linear regression analysis of lung function decline by socioeconomic factors and marital status, with adjustment for baseline lung function, age, height, smoking habits and
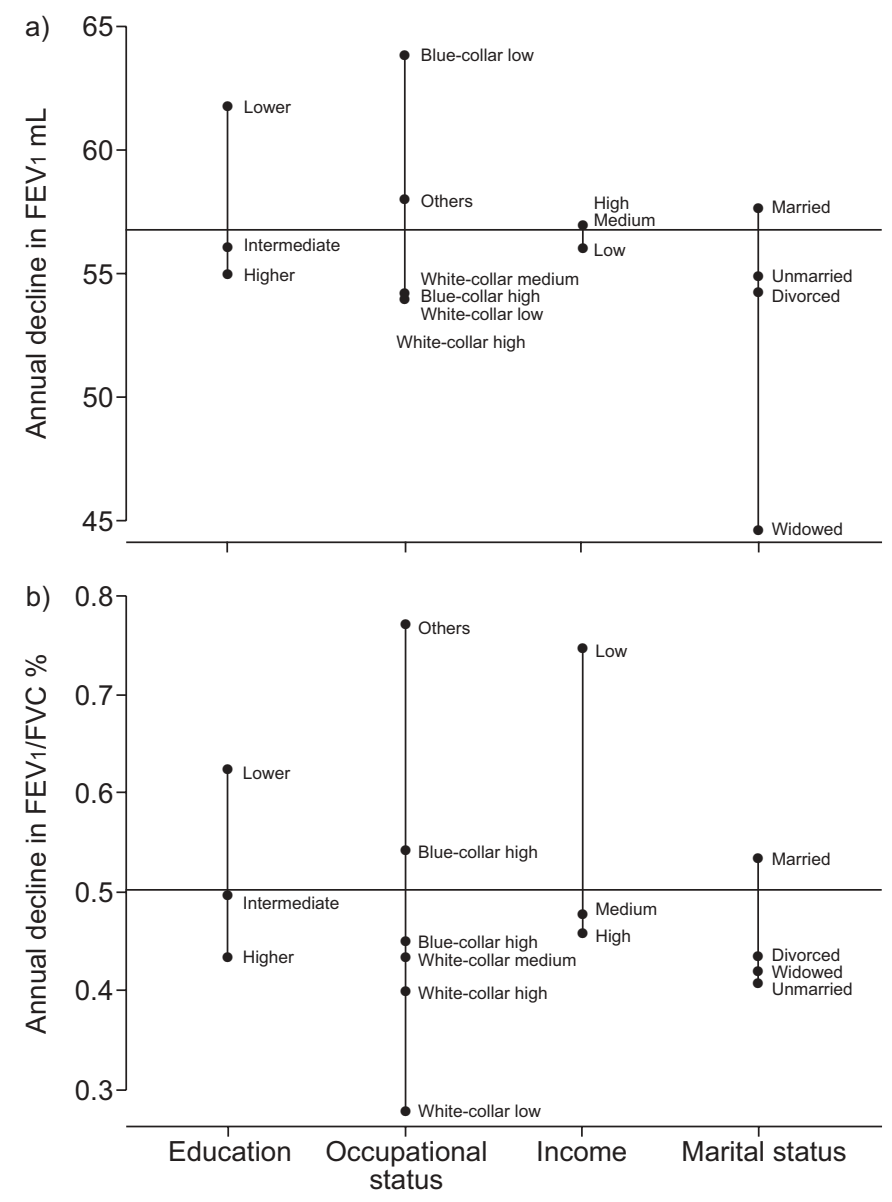

FIGURE 1. Mean annual decline in a) forced expiratory volume in $1 \mathrm{~s}$ (FEV1) and b) FEV1/forced vital capacity (FVC) among males in the Hordaland County Cohort Study 1996-1997 to 2003-2006 by educational level, occupational status, income and marital status. —_: male total population mean. $\mathrm{n}=834$. occupational exposure to dust or gas showed significant associations between lung function decline and SES for males but not females (tables 3 and 4).

Males with lower education and with blue-collar low-level occupations had 9 and $13 \mathrm{~mL}$ higher mean annual decline in FEV1 than males with white-collar high-level occupations, respectively ( $\mathrm{p}$-values 0.05 and $<0.01$, respectively). With regard to FVC, males with lower education and blue-collar low-level occupations had $19 \mathrm{~mL}$ and $13 \mathrm{~mL}$ more mean annual decline than males with higher education and whitecollar high-level occupations, respectively ( $\mathrm{p}$-values $<0.01$ and 0.02 , respectively).

Compared with unmarried females, married and widowed females had $8 \mathrm{~mL}$ and $16 \mathrm{~mL}$ more mean annual decline in FEV1, respectively (p-values 0.02 and $<0.01$, respectively; table 4). Also with FVC, married and widowed females had $7 \mathrm{~mL}$ and $16 \mathrm{~mL}$ more mean annual decline than their unmarried counterparts (p-values 0.06 and 0.01 , respectively).

\section{DISCUSSION}

The present study examined sex-specific associations between different aspects of SES and lung function in a general adult population. We found that low SES was associated with increased lung function decline in males. For females, marital status was more important than SES.

The main strengths of our study were a longitudinal study design and a large sample size, allowing for multivariate analyses of changes in lung function over time. Furthermore, we do not have reason to suspect nonresponse bias or that the estimates will not be representative for the general population. A previous study of the same cohort [20] has shown that although there were more middle-aged subjects in paid employment among responders in 1996-1997 than among nonresponders, they did not differ with regard to prevalence of respiratory symptoms. Furthermore, from baseline to followup the annual decline in FEV1 was $52 \mathrm{~mL}$ in this study population ( $48 \mathrm{~mL}$ in females and $57 \mathrm{~mL}$ in males). This is in accordance with another study from the same geographical area, where the annual decline in FEV1 was on average $53 \mathrm{~mL}$ in a general male population [21].

A limitation of our present study was loss to follow-up. As many as 25\% of the participants in 1996-1997 who were invited to the follow-up examination in 2003-2006 did not participate. Baseline lung function, age, height, occupational exposure to dust or gas, or smoking habits were not associated with loss to follow-up. Significant predictors for loss to follow-up were low educational level and low income for females, and low income and being married or divorced for males (table E1 of supplementary material). Interestingly, this was quite the opposite of the analyses in tables 3 and 4, where SES predicted accelerated lung function decline for males but not females. One could speculate that, in general, healthier people are more likely to participate in follow-up studies of lung function. Perhaps if all the invited subjects in this study had participated in the follow-up study, SES would have predicted lung function decline with comparable significance in both males and females. 

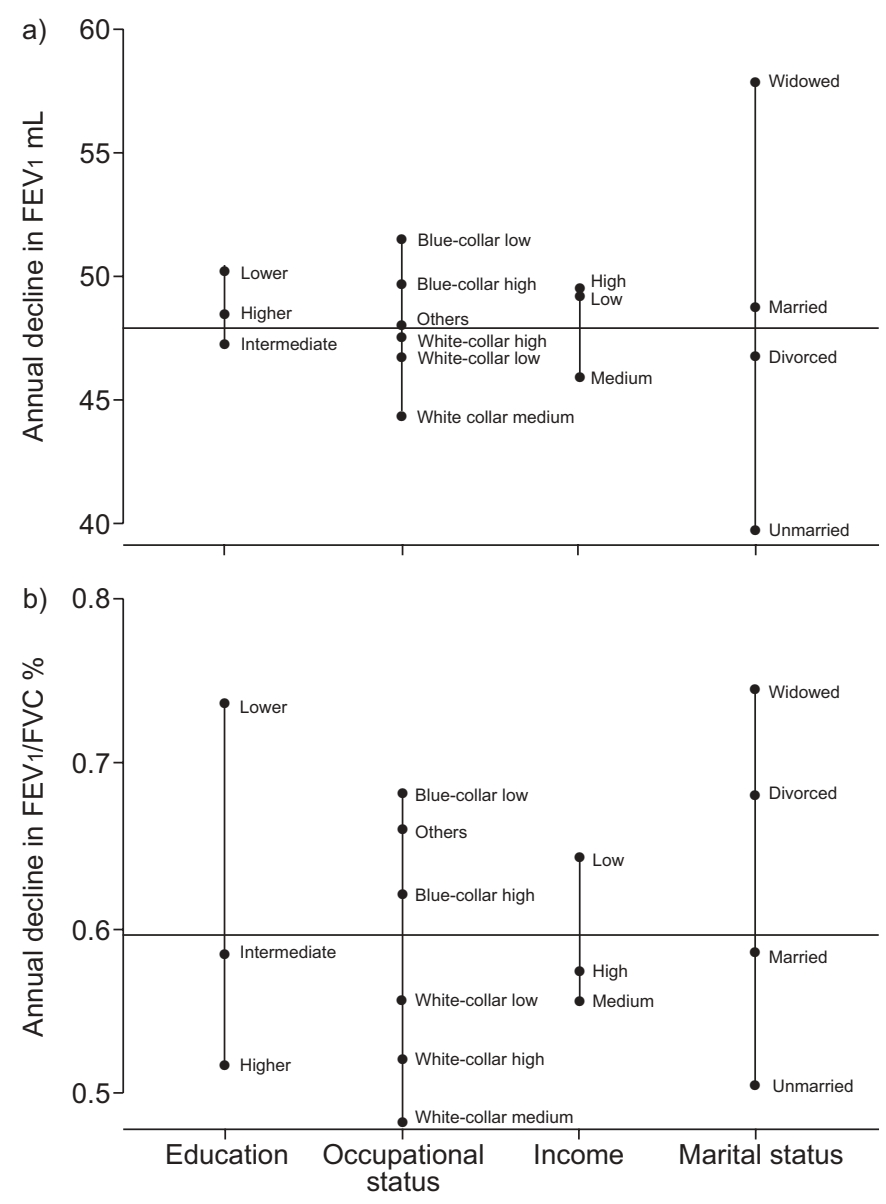

FIGURE 2. Mean annual decline in a) forced expiratory volume in $1 \mathrm{~s}$ (FEV1) and b) FEV 1 /forced vital capacity (FVC) among females in the Hordaland County Cohort Study 1996-1997 to 2003-2006 by educational level, occupational status, income and marital status. —_: female total population mean. $\mathrm{n}=810$.

A limitation of most studies focusing on repeated measures is the potential pitfall of regression to the mean. In general, when observing repeated measurements in the same subject, relatively high (or relatively low) observations are likely to be followed by less extreme ones nearer the subject's true mean [22]. The effect of regression to the mean increases with larger measurement variability. One way to reduce this problem is to use two or more baseline measurements. In our study, lung function both at baseline and follow-up was measured three times, and measurement variability at both points in time were controlled using the ATS spirometry criteria ensuring a reproducibility of the two highest measurements within $200 \mathrm{~mL}$ [23]. To further check for presence of regression to the mean, we initially plotted lung function decline against baseline lung function (results not shown). There was not markedly more decline at the tails of the baseline measurements than in the middle, suggesting that regression to the mean was not an important problem in our study.

The adjusted association between low SES and decline in lung function for males confirms the results from previous studies $[2,7-9,18,24,25]$. However, most of these studies have been cross-sectional. One of the few longitudinal studies that have examined associations between low SES and lung function decline over time is the American Coronary Artery Risk Development in Young Adults (CARDIA) study [6]. The authors observed that for subjects aged 18-30 yrs at baseline, pulmonary function declined earlier and faster for individuals with low childhood SES than for individuals with high childhood SES [6]. Similarly, in a study of middle-aged Japanese-American males from the Honolulu Heart Program, BURCHFIEL et al. [8] found that nonsmokers with lower education experienced more rapid decline in FEV1 than nonsmokers with higher education [8].

The associations between SES and lung function decline could be confounded by smoking. Smoking could also affect the observed sex differences in the present study. During the last 50 yrs, smoking has migrated its way down the SES categories from being a fashionable habit for the wealthy to becoming a working class habit. Smoking has also changed across sexes, from being a predominantly male habit to increasingly include females [26]. However, in the present study, we adjusted all multivariate analyses for smoking habits from baseline to follow-up, thus minimising the danger of confounding by smoking. Interaction analyses showed no significant interactions between marital status and smoking, between educational level and smoking, or between occupational status and smoking for any sex. For females, there was no significant interaction between income and smoking either, but for males, smokers with low income had a larger decline in FEV1/FVC than smokers with high income. However, income was the SES predictor that was not associated with lung function decline in the main analyses (table 3). Thus, this finding does not imply that the associations between SES and lung function decline is mediated by the powerful effect of smoking on lung function.

Supplemental analyses (tables E6 and E7 of online supplementary material) showed associations between cigarette smoking and FEV1 and FEV1/FVC, but not between smoking and FVC. Although smoking is a predictor for both restrictive and obstructive pulmonary disease, it is by far a more important predictor for obstruction than restriction. FEV1 is more sensitive to obstruction than FVC, and consequently the ratio is also affected by smoking.

One could hypothesise that the effect of occupational status on lung function decline was mediated by occupational exposure to airborne agents. One would perhaps expect jobs with lower status to be more prone to occupational exposure to dust or gas. However, although the two parameters are associated with each other, interaction analyses performed initially showed no interaction between exposure and status on decline in FEV1, FVC or FEV1/FVC for any sex.

Of the three SES aspects, income was only significantly associated with male FEV1/FVC decline, not with FEV1 or FVC separately. This is in contrast with what certain previous studies have found [27]. Differences in income are relatively small in Norway compared with many other countries. In the present study, baseline income for males varied from 130,000 Norwegian kroner $(\sim 14,300 €)$ on the 10 th percentile to 450,000 Norwegian kroner $(49,400 €)$ on the 90 th percentile (results not shown). However, the weak association between income and pulmonary morbidity may also be explained by other 
TABLE 3 Linear regression analyses of annual decline in forced expiratory volume in $1 \mathrm{~s}$ (FEV 1 ), forced vital capacity (FVC) and FEV1/FVC among males in the Hordaland County Cohort Study 1996-1997 to 2003-2006 by baseline educational level, occupational status, income and marital status

FEV1 decline $\mathrm{mL}$

$\begin{array}{lc}\text { Educational level } & \\ \text { Higher education } & 0 \\ \text { Intermediate education } & 2.1(0.519) \\ \text { Lower education } & 9.4(0.048)^{*} \\ \text { Occupational status } & \\ \text { White-collar high level } & 0 \\ \text { White-collar medium level } & -0.7(0.880) \\ \text { White-collar low level } & -0.3(0.970) \\ \text { Blue-collar high level } & 1.8(0.672) \\ \text { Blue-collar low level } & 13.3(0.002) \\ \text { Others } & 3.8(0.462) \\ \text { Income } & \\ \text { High income } & 0 \\ \text { Medium income } & -0.8(0.806) \\ \text { Low income } & -0.2(0.969) \\ \text { Marital status } & \\ \text { Unmarried } & 0 \\ \text { Married } & -0.1(0.972) \\ \text { Widowed } & -12.4(0.420) \\ \text { Divorced } & -5.0(0.397)\end{array}$

FVC decline $\mathrm{mL}$

FEV $1 /$ FVC decline \%

Data are presented as parameter estimate ( $p$-value), unless otherwise indicated. All analyses are adjusted for baseline lung function, age, height, occupational exposure to dust or gas, and smoking habits from baseline to follow-up. *: $p<0.05 . n=834$.

TABLE 4 Linear regression analyses of annual decline in forced expiratory volume in $1 \mathrm{~s}$ (FEV1), forced vital capacity (FVC) and FEV1/FVC among females in the Hordaland County Cohort Study 1996-1997 to 2003-2006 by baseline educational level, occupational status, income and marital status

FEV1 decline $\mathrm{mL}$

\section{Educational level}

Higher education

Intermediate education

Lower education

\section{Occupational status}

White-collar high level

White-collar medium level

White-collar low level

Blue-collar high level

Blue-collar low level

Others

\section{Income}

High income

Medium income

Low income

\section{Marital status}

Unmarried

Married

Widowed

Divorced

\section{0}

$-2.6(0.319)$

$1.0(0.782)$

0

$-3.9(0.468)$

$-0.6(0.909)$

$3.6(0.697)$

$3.1(0.551)$

$-3.3(0.557)$

0

$-2.3(0.430)$

$-0.8(0.799)$

0

$7.8(0.017)^{*}$

$15.5(0.004)^{*}$

$5.0(0.262)$
FVC decline $\mathrm{mL}$

0

$-3.0(0.314)$

$-1.3(0.750)$

0

$-0.6(0.917)$

$0.4(0.954)$

$1.4(0.898)$

$3.2(0.599)$

$1.2(0.855)$

\section{0}

$-0.5(0.876)$

$0.0(0.995)$

0

$7.0(0.059)$

$16.2(0.009)^{*}$

$3.0(0.560)$
FEV $1 /$ FVC decline $\%$

Data are presented as parameter estimate ( $\mathrm{p}$-value), unless otherwise indicated. All analyses are adjusted for baseline lung function, age, height, occupational exposure to dust or gas, and smoking habits from baseline to follow-up. *: $p<0.05 . n=810$ 
mechanisms than egalitarianism. Results from the British Household Panel Survey suggest that economics matters less than, for instance, marital status and psychological distress with regard to longevity [28].

In this study population, SES affected lung function among males but not females. Several studies have shown that SES is a significant predictor for impaired lung function for both males and females $[2,7]$. However, it has also been shown that although SES is a significant risk factor for disease among both sexes, low individual SES affects males more than females [18, 29]. A possible explanation for the lack of association between SES and female lung function in our study could be that the females' SES will be linked to their husband's social position, at least among the elderly [29]. Information on household income rather than personal income, and information on partner's education and occupation could shed some light on this hypothesis.

Another noteworthy result from the present study was that married females and widows had a larger lung function decline than unmarried females, even after adjusting for all potential confounders including age. One could hypothesise that a nonlinear association between lung function decline and age could be a confounder for the results in the present study, since the age adjustment performed here was linear. The estimates for the youngest (unmarried females) and oldest (widows) could have been affected by a linear age adjustment to give an unrealistically minimised lung function decline for the unmarried and a corresponding maximised lung function decline for the widows. However, additional analyses where we adjusted for age $\mathrm{e}^{2}$ did not alter any of the estimates (results not shown).

The significant association between marital status and female lung function decline was based on marital status in 19961997. Perhaps the results would be affected by taking into consideration those who changed their marital status during the study period. To examine this possibility further, we performed additional analyses where we included changes in marital status from baseline to follow-up instead of simple baseline marital status. This did not change any of the results (results not shown).

The finding that married females had larger lung function decline than unmarried females contrasts with results from previous studies, showing that marriage has a beneficial health effect on for instance physical functioning and hospital admissions [30, 31]. However, previous research is not entirely consistent; a Dutch study showed that adults who had never been married had lower healthcare utilisation than those who were married [32]. Furthermore, JounG et al. [33] reported from the GLOBE study that people who live alone have higher morbidity rates than those who live with a partner. Following this line of reasoning, a high degree of unmarried cohabitation could have influenced our results. This would potentially affect the younger age segments, where unmarried cohabitation is more common. Unfortunately, we do not have information in the present study on the proportions of unmarried females in the study population who were single or cohabitants. An alternative explanation for the negative marriage effect could be passive smoking, since females have traditionally experienced more passive smoking than males. However, additional analyses where we adjusted for passive smoking reported at baseline did not change the marital status estimates (results not shown).

Finally, to further investigate the associations between SES, marital status and lung function, we also performed alternative multivariate analyses with baseline lung function and followup lung function as outcomes instead of lung function decline (tables E2-E5 in the online supplementary material). This was done to check if associations remained the same in a crosssectional perspective. Interestingly, the results were mainly the same for males, but altered somewhat for females. Low occupational status was a significant predictor for low lung function in the cross-sectional analyses. Being married was associated with lower FEV1/FVC but not FEV1 and FVC. These findings may suggest that marital status in an epidemiological perspective gains importance with increasing time, and may be a "slow-acting" risk factor for female pulmonary health, but more studies are needed to elaborate on this.

To conclude, our study confirms the notion that SES is an independent and important risk factor for decline in lung function, at least among males. Future studies should examine more closely the associations between SES and marital status, and pulmonary health among females. The "healthy marriage effect" may have exceptions, especially among females.

\section{SUPPORT STATEMENT}

The work was supported by the Norwegian Research Council, GlaxoSmithKline and the Foundation for Respiratory Research at Haukeland University Hospital in Bergen, Norway.

\section{STATEMENT OF INTEREST}

A statement of interest for P.S. Bakke can be found at www.erj. ersjournals.com $/ \mathrm{misc} /$ statements.dtl

\section{ACKNOWLEDGEMENTS}

The authors thank statistician R. Miodini Nilsen for valuable methodological advice.

\section{REFERENCES}

1 Naess O, Claussen B, Thelle DS, et al. Four indicators of socioeconomic position: relative ranking across causes of death. Scand J Public Health 2005; 33: 215-221.

2 Prescott E, Lange P, Vestbo J. Socioeconomic status, lung function and admission to hospital for COPD: results from the Copenhagen City Heart Study. Eur Respir J 1999; 13: 1109-1114.

3 Marmot MG, McDowall ME. Mortality decline and widening social inequalities. Lancet 1986; 2: 274-276.

4 Celli BR, MacNee W. Standards for the diagnosis and treatment of patients with COPD: a summary of the ATS/ERS position paper. Eur Respir J 2004; 23: 932-946.

5 McFadden E, Luben R, Wareham N, et al. How far can we explain the social class differential in respiratory function? A crosssectional population study of 21,991 men and women from EPICNorfolk. Eur J Epidemiol 2009; 24: 193-201.

6 Jackson B, Kubzansky LD, Cohen S, et al. A matter of life and breath: childhood socioeconomic status is related to young adult pulmonary function in the CARDIA study. Int J Epidemiol 2004; 33: 271-278. 
7 Shohaimi S, Welch A, Bingham S, et al. Area deprivation predicts lung function independently of education and social class. Eur Respir J 2004; 24: 157-161.

8 Burchfiel CM, Marcus EB, Sharp DS, et al. Characteristics associated with rapid decline in forced expiratory volume. Ann Epidemiol 1996; 6: 217-227.

9 Hole DJ, Watt GC, Davey-Smith G, et al. Impaired lung function and mortality risk in men and women: findings from the Renfrew and Paisley prospective population study. BMJ 1996; 313: 711-715.

10 Hegewald MJ, Crapo RO. Socioeconomic status and lung function. Chest 2007; 132: 1608-1614.

11 Mannino DM, Buist AS. Global burden of COPD: risk factors, prevalence, and future trends. Lancet 2007; 370: 765-773.

12 Prescott E, Vestbo J. Socioeconomic status and chronic obstructive pulmonary disease. Thorax 1999; 54: 737-741.

13 Liberatos P, Link BG, Kelsey JL. The measurement of social class in epidemiology. Epidemiol Rev 1988; 10: 87-121.

14 Jaffe DH, Manor O, Eisenbach Z, et al. The protective effect of marriage on mortality in a dynamic society. Ann Epidemiol 2007; 17: 540-547.

15 Gulsvik A, Humerfelt S, Bakke P, et al. Norwegian population surveys on respiratory health in adults: objectives, design, methods, quality controls and response rates. Clin Resp J 2008; 2 $10-26$.

16 Johannessen A, Lehmann S, Omenaas ER, et al. Post-bronchodilator spirometry reference values in adults and implications for disease management. Am J Respir Crit Care Med 2006; 173: 1316-1325.

17 Eagan TM, Gulsvik A, Eide GE, et al. Occupational airborne exposure and the incidence of respiratory symptoms and asthma. Am J Respir Crit Care Med 2002; 166: 933-938.

18 Bakke PS, Hanoa R, Gulsvik A. Educational level and obstructive lung disease given smoking habits and occupational airborne exposure: a Norwegian community study. Am J Epidemiol 1995; 141: 1080-1088.

19 Eriksson R, Goldthorpe J. The Constant Flux. Oxford, Oxford University Press, 1999.
20 Eagan TM, Eide GE, Gulsvik A, et al. Nonresponse in a community cohort study: predictors and consequences for exposure-disease associations. J Clin Epidemiol 2002; 55: 775-781.

21 Humerfelt S, Gulsvik A, Skjaerven R, et al. Decline in FEV1 and airflow limitation related to occupational exposures in men of an urban community. Eur Respir J 1993; 6: 1095-1103.

22 Barnett AG, van der Pols JC, Dobson AJ. Regression to the mean: what it is and how to deal with it. Int J Epidemiol 2005; 34: 215-220.

23 American Thoracic Society, Standardization of Spirometry, 1994 Update. American Thoracic Society. Am J Respir Crit Care Med 1995; 152: 1107-1136.

24 Eagan TM, Gulsvik A, Eide GE, et al. The effect of educational level on the incidence of asthma and respiratory symptoms. Respir Med 2004; 98: 730-736.

25 Cerveri I, Accordini S, Corsico A, et al. Chronic cough and phlegm in young adults. Eur Respir J 2003; 22: 413-417.

26 Mannino DM. COPD: epidemiology, prevalence, morbidity and mortality, and disease heterogeneity. Chest 2002; 121: 121S-126S.

27 Lemstra M, Neudorf C, Opondo J. Health disparity by neighbourhood income. Can J Public Health 2006; 97: 435-439.

28 Gardner J, Oswald A. How is mortality affected by money, marriage, and stress? J Health Econ 2004; 23: 1181-1207.

29 Koskinen S, Martelin T. Why are socioeconomic mortality differences smaller among women than among men? Soc Sci Med 1994; 38: 1385-1396.

30 Wong AW, Gan WQ, Burns J, et al. Acute exacerbation of chronic obstructive pulmonary disease: influence of social factors in determining length of hospital stay and readmission rates. Can Respir J 2008; 15: 361-364.

31 Mackenbach JP, Borsboom GJ, Nusselder WJ, et al. Determinants of levels and changes of physical functioning in chronically ill persons: results from the GLOBE Study. J Epidemiol Community Health 2001; 55: 631-638.

32 Joung IM, van der Meer JB, Mackenbach JP. Marital status and health care utilization. Int J Epidemiol 1995; 24: 569-575.

33 Joung IM, van de Mheen H, Stronks $\mathrm{K}$, et al. Differences in selfreported morbidity by marital status and by living arrangement. Int J Epidemiol 1994; 23: 91-97. 